\title{
Non-volatile resistive switching in the Mott insulator $\left(\mathrm{V}_{1-\mathrm{x}} \mathrm{Cr}_{\mathrm{x}}\right)_{2} \mathrm{O}_{3}$
}

\author{
M. Querré ${ }^{1,2}$, J. Tranchant ${ }^{1}$, B. Corraze ${ }^{1}$, S. Cordier ${ }^{2}$, V. Bouquet $^{2}$, \\ S. Députier ${ }^{2}$, M. Guilloux-Viry ${ }^{2}$, M.-P. Besland ${ }^{1}$, E. Janod ${ }^{1}$ and L. Cario ${ }^{1}$ \\ 1 Institut des Matériaux Jean Rouxel (IMN), Université de Nantes, UMR CNRS 6502, 44322 Nantes (FRANCE) \\ 2 Institut des Sciences Chimiques de Rennes (ISCR), Université de Rennes 1, UMR CNRS 6226, 35042 Rennes (FRANCE) \\ E-mail : Laurent.Cario@cnrs-imn.fr
}

\begin{abstract}
The discovery of non-volatile resistive switching in Mott insulators related to an electric-field-induced insulator to metal transition (IMT) has paved the way for their use in a new type of non-volatile memories, the Mott memories. While most of the previous studies were dedicated to uncover the resistive switching mechanism and explore the memory potential of chalcogenide Mott insulators, we present here a comprehensive study of resistive switching in the canonical oxide Mott insulator $\left(\mathrm{V}_{1-\mathrm{x}} \mathrm{Cr}_{\mathrm{x}}\right)_{2} \mathrm{O}_{3}$. Our work demonstrates that this compound undergoes a non-volatile resistive switching under electric field. This resistive switching is induced by a Mott transition at the local scale which creates metallic domains closely related to existing phases of the temperature-pressure phase diagram of $\left(\mathrm{V}_{1-\mathrm{x}} \mathrm{Cr}_{\mathrm{x}}\right)_{2} \mathrm{O}_{3}$. Our work demonstrates also reversible resistive switching in $\left(\mathrm{V}_{1-\mathrm{x}} \mathrm{Cr}_{\mathrm{x}}\right)_{2} \mathrm{O}_{3}$ crystals and thin film devices. Preliminary performances obtained on $880 \mathrm{~nm}$ thick layers with $500 \mathrm{~nm}$ electrodes show the strong potential of Mott memories based on the Mott insulator $\left(\mathrm{V}_{1-\mathrm{x}} \mathrm{Cr}_{\mathrm{x}}\right)_{2} \mathrm{O}_{3}$.
\end{abstract}

Keywords: Mott insulators, $\mathrm{V}_{2} \mathrm{O}_{3}$, Mott transition, resistive switching, non-volatile memory, ReRAM.

\section{Introduction}

Resistive Random Access Memories (ReRAM) are considered as one of the most promising technologies for future non-volatile memories. They are based on resistive switching mainly related to two physical mechanisms: ionic electromigration (anionic for OxRAM and cationic for CBRAM) and thermochemical reactions (typically in $\mathrm{NiO}$ ) [1, 2, 3]. But recently the insulator to metal transition (IMT) in Mott insulators has focused much attention due to its serious potential as an alternative mechanism for ReRAM [4]. Mott insulating state can be usually destabilized by pressure and doping into a correlated metal phase via an isostructural electronic phase transition, as shown for example in the temperature-pressure phase diagram of $\mathrm{V}_{2} \mathrm{O}_{3}$ in Fig. 1(a) [5]. However recent works $[4,6,7,8]$ have shown that electric field can also destabilize the Mott insulating state and trigger a non-volatile resistive switching in these materials. Studies performed on the chalcogenide compounds $\mathrm{AM}_{4} \mathrm{Q}_{8}(\mathrm{~A}=\mathrm{Ga}$, Ge; $M=N b, V, T a ; Q=S$, Se) have demonstrated that this resistive switching (RS) originates from an avalanche breakdown phenomenon [7] which causes the collapse of the paramagnetic Mott insulator state at the nanoscale [10] and results in the creation of granular conductive filaments [11].

This discovery on the chalcogenide $\mathrm{AM}_{4} \mathrm{Q}_{8}$ compounds raises the question of the universal nature of this new type of non-volatile resistive switching. We have therefore sought such resistive switching in the most famous canonical narrow gap Mott insulator, namely $\left(\mathrm{V}_{1-\mathrm{x}} \mathrm{Cr}_{\mathrm{x}}\right)_{2} \mathrm{O}_{3}$. We report here that this oxide Mott insulator exhibits a non-volatile resistive switching of the same physical nature as the $\mathrm{AM}_{4} \mathrm{Q}_{8}$ compounds. Moreover we show that the performances of a $\left(\mathrm{V}_{1-\mathrm{x}} \mathrm{Cr}_{\mathrm{x}}\right)_{2} \mathrm{O}_{3}$ memory cell obtained on a device mostly compatible with microelectronics standards are very promising.

\section{Material and methods}

\section{1. $\left(\mathrm{V}_{1-\mathrm{x}} \mathrm{Cr}_{\mathrm{x}}\right)_{2} \mathrm{O}_{3}$ crystals synthesis}

$\mathrm{V}_{2} \mathrm{O}_{5}$ (Aldrich, > $99.6 \%$ ) powder was first dried at $400^{\circ} \mathrm{C}$, then mixed with $\mathrm{Cr}_{2} \mathrm{O}_{3}$ (Prolabo, $99.9 \%$ ) in the $97.5: 2.5$ molar ratio, and then heat-treated at $900^{\circ} \mathrm{C}$ for $10 \mathrm{~h}$ under a $95 \% \mathrm{Ar}-5 \% \mathrm{H}_{2}$ gas flow. Half a gram of the obtained $\left(\mathrm{V}_{0.975} \mathrm{Cr}_{0.025}\right)_{2} \mathrm{O}_{3}$ powder was then placed in a silica tube together with $40 \mathrm{mg}$ of sulfur as a vapor phase transport agent. The tube was vacuum sealed, heated up to $1050^{\circ} \mathrm{C}$ in a temperature gradient $\left(\approx 10^{\circ} \mathrm{C} / \mathrm{cm}\right)$ furnace and then cooled down at $-2^{\circ} \mathrm{C} / \mathrm{h}$ to $900^{\circ} \mathrm{C}$, before a faster cooling $\left(-300^{\circ} \mathrm{C} / \mathrm{h}\right)$ to room temperature. Energy Dispersive X-ray Spectroscopy analyses carried out on the obtained crystals (typical size $100 \times$ $50 \times 300 \mu \mathrm{m})$ indicate that the $\mathrm{V} / \mathrm{Cr}$ ratio is close to the nominal value and show the absence of sulfur impurities in the crystals.

\subsection{Thin film synthesis and device preparation}

Thin films of $\left(\mathrm{V}_{0.95} \mathrm{Cr}_{0.05}\right)_{2} \mathrm{O}_{3}$ were deposited by reactive co-sputtering of $\mathrm{V}$ and $\mathrm{Cr}$ targets in $\mathrm{Ar} / \mathrm{O}_{2}$ discharge [12], leading to thicknesses in the $100-880 \mathrm{~nm}$ range. Post deposition annealing at $500^{\circ} \mathrm{C}$ in a reducing atmosphere yields crystallized and single-phased $\left(\mathrm{V}_{0.95} \mathrm{Cr}_{0.05}\right)_{2} \mathrm{O}_{3}$ layers as checked by X-Ray diffraction (XRD). In $\mathrm{V}_{2} \mathrm{O}_{3}$, deviation from the ideal V/O ratio by $1.75 \%$ lowers the magnetic ordering temperature (paramagnetic metal PM to antiferromagnetic insulator AFI transition, see Fig. 1(a)) from $155 \mathrm{~K}$ to $11 \mathrm{~K}$ [13]. But our $\left(\mathrm{V}_{0.95} \mathrm{Cr}_{0.05}\right)_{2} \mathrm{O}_{3}$ thin films deposited on $\mathrm{SiO}_{2} / \mathrm{Si}$ substrates and measured with four probes in a planar configuration exhibit a paramagnetic Mott insulator PMI - AFI transition temperature (158 K) very close to the expected one (175K), which indicates that they are almost stoichiometric. This prevents spurious electromigration processes associated to ionic diffusion and excludes potential OxRAM/CBRAM mechanisms. Devices based on a symetric TiN / $\left(\mathrm{V}_{0.95} \mathrm{Cr}_{0.05}\right)_{2} \mathrm{O}_{3} / \mathrm{TiN}$ MIM structure were subsequently realized with via diameters in the 330 - $1600 \mathrm{~nm}$ range.

\subsection{Electrical measurements}

$\left(\mathrm{V}_{0.975} \mathrm{Cr}_{0.025}\right)_{2} \mathrm{O}_{3}$ crystals were contacted with $17 \mu \mathrm{m}$ gold wires using a carbon paste (Electrodag PR-406) and then annealed in vacuum at $150{ }^{\circ} \mathrm{C}$ for 20 min to achieve a low contact resistance. The low-bias resistance was measured 
using a high-impedance Keithley 236 source-measure unit. Thin film devices and crystals were connected in series with a load resistance $\mathrm{R}_{\text {load }}$ playing the role of current limiter and ranging in the $10-80 \%$ of the device or crystal resistance. The write-erase cycling measurements were performed using an Agilent 81150A pulse generator.

\section{Results and Discussion}

\subsection{Resistive switching in $\left(V_{1-x} C r_{x}\right)_{2} O_{3}$ crystals}

Figure 1(b) shows the resistance versus temperature (blue curve) measured on a $\left(\mathrm{V}_{0.975} \mathrm{Cr}_{0.025}\right)_{2} \mathrm{O}_{3}$ single crystal grown by the sulfur vapor transport method. Above 190K, the crystal shows an insulating behavior consistent with the expected PMI state. The transition from the PMI to the AFI phase occurs around $180 \mathrm{~K}$ which is again in good agreement with the value expected from the phase diagram (see Figure 1(a)). The crystal in series with a load resistance was subsequently subjected to an electric pulse $(75 \mathrm{~V}, 100 \mu$ s at $155 \mathrm{~K})$. As shown in Figure 1(b), the application of this electric pulse induces a non-volatile decrease of the resistance $R(T)$ by several orders of magnitude at low temperature (red curve). This observation reveals that the application of short electric pulses can induce a non-volatile metal-insulator transition in the Mott insulator $\left(\mathrm{V}_{0.975} \mathrm{Cr}_{0.025}\right)_{2} \mathrm{O}_{3}$ which is reminiscent of the behavior of $\mathrm{AM}_{4} \mathrm{Q}_{8}$ compounds [14].

Previous in-depth studies of $\mathrm{GaTa}_{4} \mathrm{Se}_{8}$ have unveiled important features of the non-volatile resistive switching of the $\mathrm{AM}_{4} \mathrm{Q}_{8}$ family of compounds. This narrow gap Mott insulator exhibits indeed an insulator to metal transition under pressure (at about $5 \mathrm{GPa}$ ) and becomes superconducting below $T_{C}=4-7 \mathrm{~K}$ above $11 \mathrm{GPa}$ [11,12]. In $\mathrm{GaTa}_{4} \mathrm{Se}_{8}$, Scanning Tunneling Microscopy (STM) measurements have revealed that the non-volatile resistive switching is related to an electronic phase separation at the nanoscale $[10,11]$. The surface topography of pristine $\mathrm{GaTa}_{4} \mathrm{Se}_{8}$ crystals is structureless, but after resistive switching some granular filamentary structures qualitatively oriented along the direction of the electric pulses appear on the surface. Spectroscopic (STS) experiments have confirmed the existence of electric-field-induced metallic nano-domains of about 30-70 nm within these granular filamentary paths. Moreover the presence of granular superconductivity at low temperature, reminiscent of the one observed under pressure, directly suggests the presence of compressed metallic nano-domains in transited crystals of $\mathrm{GaTa}_{4} \mathrm{Se}_{8}$ [11].

This hypothesis was further tested in the case of $\left(\mathrm{V}_{1-\mathrm{x}} \mathrm{Cr}_{\mathrm{x}}\right)_{2} \mathrm{O}_{3}$ using the transition towards the AFI phase as a probe. The phase diagram of this compound (see Fig. 1a) shows indeed that the transition temperature from the Mott insulating phase (PMI) to the AFI phase does not change much with composition or pressure. Conversely the huge variation observed under composition/pressure for the transition temperature from the metallic (PM) to the AFI phase may be used to characterized the formation under electric pulse of compressed metallic domains. Figure 1(b) compare the $R(T)$ curves of the $\left(\mathrm{V}_{0.975} \mathrm{Cr}_{0.025}\right)_{2} \mathrm{O}_{3}$ crystal in the pristine state (blue curve) and after resistive switching (red curve). Both curves show the signature of the PMI-AFI transition around 180K; in the switched crystal, it corresponds to the transition of the $\left(\mathrm{V}_{0.975} \mathrm{Cr}_{0.025}\right)_{2} \mathrm{O}_{3}$ matrix embedding the filamentary
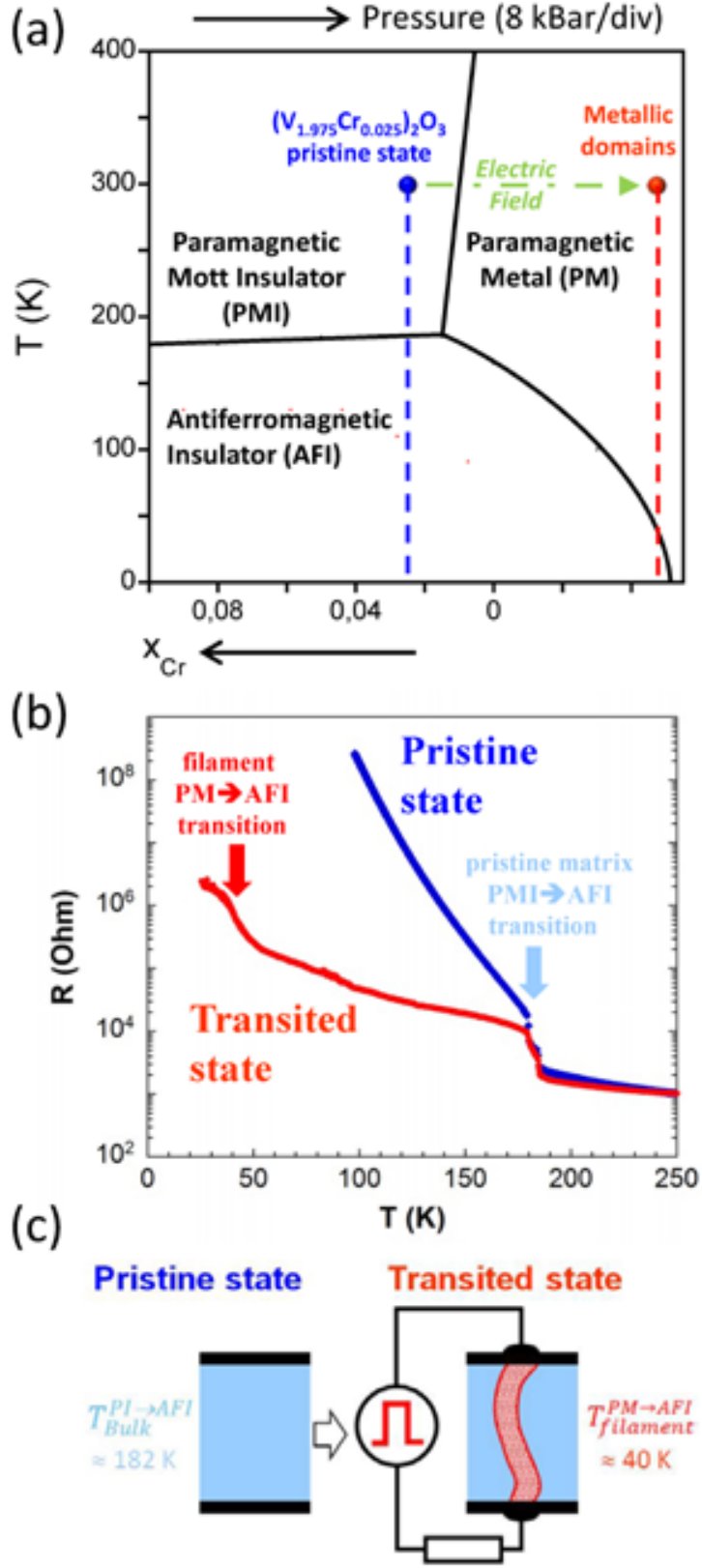

Fig. 1 : (a) Theoretical phase diagram of $\left(\mathrm{V}_{1-\mathrm{x}} \mathrm{Cr}_{\mathrm{x}}\right)_{2} \mathrm{O}_{3}$ [5]. (b) Evolution of resistance vs temperature of a $\left(\mathrm{V}_{0.975} \mathrm{Cr}_{0.025}\right)_{2} \mathrm{O}_{3}$ crystal in its pristine state and in a transited state. As expected the PMI-AFI phase transition occurs around $180 \mathrm{~K}$ in the pristine state (see blue dotted line in (a) and blue arrow in (b)). In the transited state a new transition occurs at $40 \mathrm{~K}$ suggesting the formation of compressed metallic domains (see red dotted line in (a) and red arrow in (b)). (c) Schematical drawing of the formation of a granular filamentary path in the transited state of a $\left(\mathrm{V}_{0.95} \mathrm{Cr}_{0.05}\right)_{2} \mathrm{O}_{3}$ crystal containing some compressed domains with a PM-AFI phase transition around $40 \mathrm{~K}$ as indicated by the red dotted line in (a).

conducting path (light blue regions in Fig.1 (c)). However a new transition is clearly observable around $40 \mathrm{~K}$ in the $R(T)$ curve of the switched $\left(\mathrm{V}_{0.975} \mathrm{Cr}_{0.025}\right)_{2} \mathrm{O}_{3}$ crystal (in red). According to the phase diagram of this system, this may be viewed as the $\mathrm{PM} \rightarrow \mathrm{AFI}$ transition of $\left(\mathrm{V}_{0.975} \mathrm{Cr}_{0.025}\right)_{2} \mathrm{O}_{3}$ under a pressure of $30 \mathrm{kbar}$ [17]. This is a clear indication of the creation of compressed metallic domains under electric field. After resistive switching the metallic domains would therefore correspond to $\left(\mathrm{V}_{0.975} \mathrm{Cr}_{0.025}\right)_{2} \mathrm{O}_{3}$ domains under pressure, as indicated by the red dotted line in Fig. 1(a). From these 


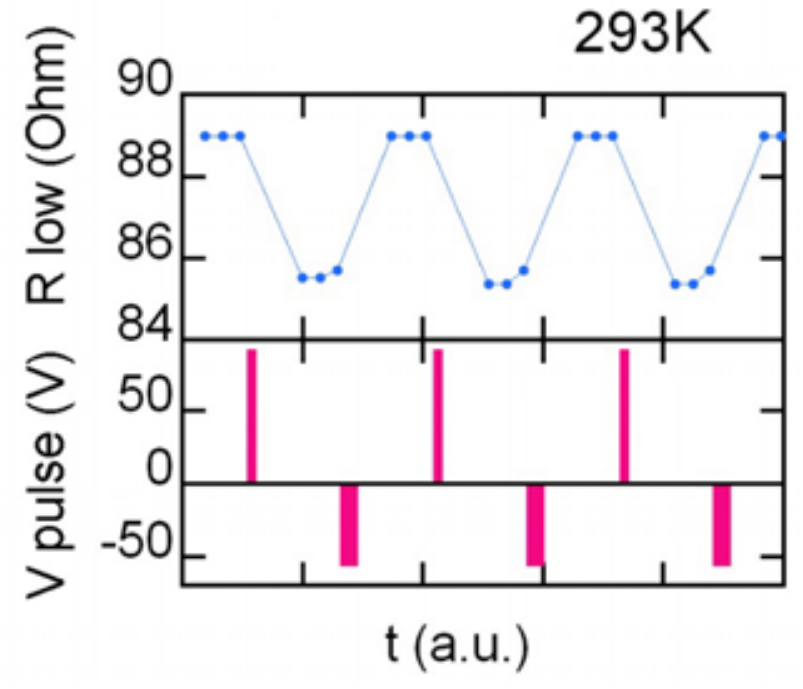

Fig. 2: Resistive switching cycles obtained at room temperature on large crystals of $\left(\mathrm{V}_{0.925} \mathrm{Cr}_{0.075}\right)_{2} \mathrm{O}_{3}$ by applying a series of pulses (+90V for the SET and -50V for RESET).

experiments, we may therefore infer, as sketched in Figure 1 (c), that the Mott insulator compound $\left(\mathrm{V}_{1-\mathrm{x}} \mathrm{Cr}_{\mathrm{x}}\right)_{2} \mathrm{O}_{3}$ that has experienced a resistive switching under electric field contains some conducting granular filamentary paths embedded in a pristine like matrix. Moreover these granular filamentary paths include some compressed regions that have undergone a Mott insulator to metal transition.

\subsection{Reversible resistive switching in $\left(\mathrm{V}_{1-\mathrm{x}} C \mathrm{Cr}_{\mathrm{x}}\right)_{2} \mathrm{O}_{3}$ crystals}

Figure 2 reveals another interesting feature of the non-volatile resistive switching in $\left(\mathrm{V}_{1-\mathrm{x}} \mathrm{Cr}_{\mathrm{x}}\right)_{2} \mathrm{O}_{3}$. It shows that reversible resistive switching may be obtained on crystal at room temperature using a series of voltage pulses $(+90 \mathrm{~V}$ for the SET and -50V for RESET). This observation is consistent with data obtained on $\mathrm{AM}_{4} \mathrm{Q}_{8}$ [6]. The RESET transition was explained on the basis of a modeling work by the transition back of the metallic domains to the insulating state thanks to a thermal activation process enabled by self-Joule heating of the filament during the pulse $[7,16]$. This reversible resistive switching opens the way to the use of the Mott insulator $\left(\mathrm{V}_{1-\mathrm{x}} \mathrm{Cr}_{\mathrm{x}}\right)_{2} \mathrm{O}_{3}$ as an active material of a non-volatile ReRAM memory. However the $\mathrm{R}_{\mathrm{OFF}} / \mathrm{R}_{\mathrm{ON}}$ ratio on crystals is quite poor ( $<1.05$ ), $\mathrm{R}_{\mathrm{OFF}}$ and $\mathrm{R}_{\mathrm{ON}}$ being defined respectively as the resistance values of high (OFF) and low (ON) resistance states. This is related to the large section of the crystal compared to the size of the conducting filamentary path embedded in this crystal. To enhance the $\mathrm{R}_{\mathrm{OFF}} / \mathrm{R}_{\mathrm{ON}}$ ratio it is therefore important to design new devices with smaller electrode areas.

\subsection{Electrical performances of $\left(\mathrm{V}_{1-x} \mathrm{Cr}_{x}\right)_{2} \mathrm{O}_{3}$ thin film devices}

To further explore the potential for memory application, some devices in Metal-Insulator-Metal (MIM) structure with small electrode areas (vias) were prepared from well crystallized thin film of $\left(\mathrm{V}_{1-\mathrm{x}} \mathrm{Cr}_{\mathrm{x}}\right)_{2} \mathrm{O}_{3}$ Mott insulators (see Figure 3).

As displayed in Fig. 4(a) in the case of a $880 \mathrm{~nm}$ thick $\left(\mathrm{V}_{0.95} \mathrm{Cr}_{0.05}\right)_{2} \mathrm{O}_{3}$ film, the application of $3.5 \mathrm{~V} / 1.5 \mu \mathrm{s}$ (SET transition from the high to the low resistance state) and $4.5 \mathrm{~V} /$
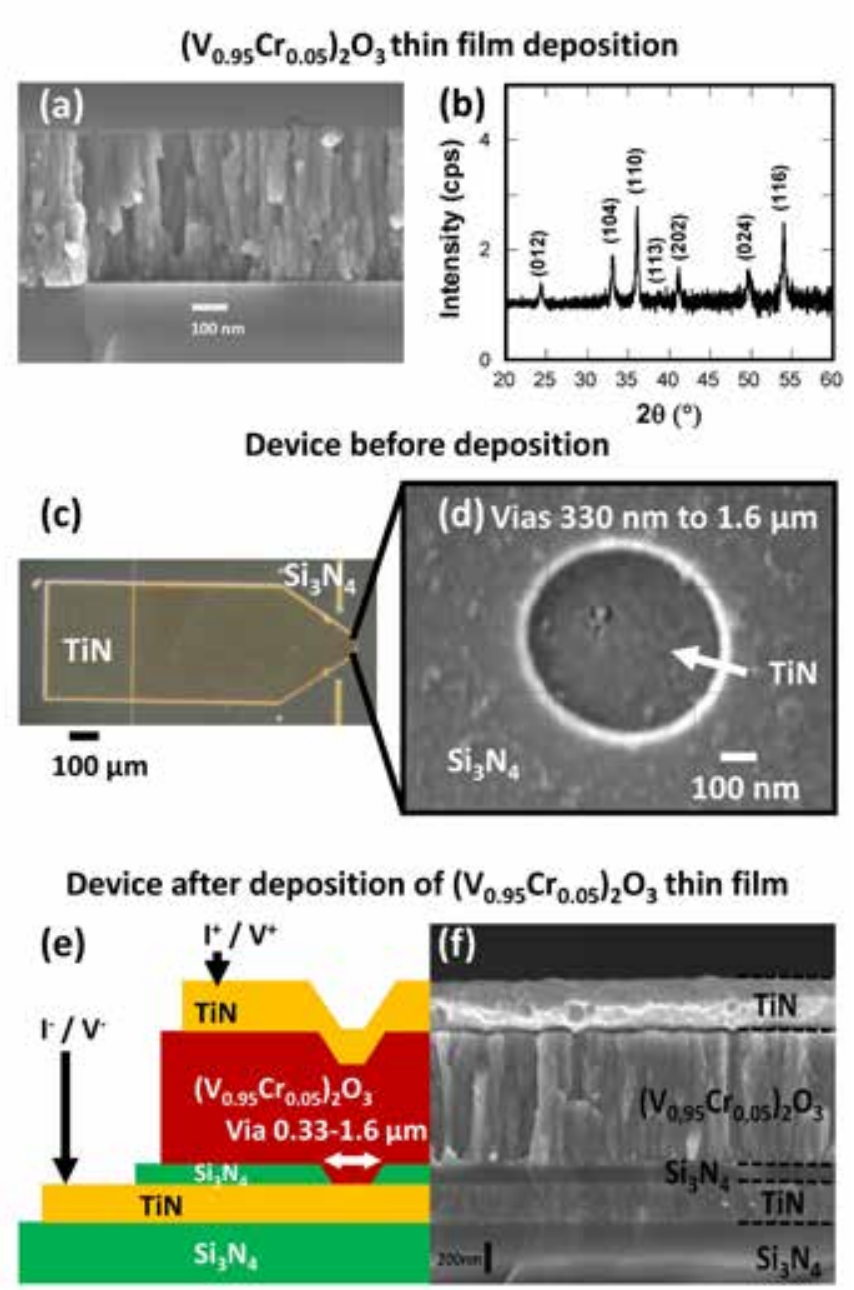

Fig. 3: (a) SEM cross-sectional picture and (b) XRD diagram of a 880 $\mathrm{nm}$ thick $\left(\mathrm{V}_{0.95} \mathrm{Cr}_{0.05}\right)_{2} \mathrm{O}_{3}$ film. (c) Top view of a substrate used for deposition of $\left(\mathrm{V}_{0.95} \mathrm{Cr}_{0.05}\right)_{2} \mathrm{O}_{3}$ thin films and (d) SEM picture of a bottom electrode with a via of $500 \mathrm{~nm}$ in diameter. (e) Cross-sectional schematic view of the device based on $\left(\mathrm{V}_{0.95} \mathrm{Cr}_{0.05}\right)_{2} \mathrm{O}_{3}$ and (f) corresponding back scattered electron SEM picture of the multi-layer.

$250 \mathrm{~ns}$ (RESET transition) pulses to such device with $500 \mathrm{~nm}$ vias engenders resistive switching cycles with an average $\mathrm{R}_{\mathrm{OFF}} / \mathrm{R}_{\mathrm{ON}}$ close to 20 . This ratio evolves linearly with the inverse of the electrode surface $1 / \mathrm{S}$, since vias of $330 \mathrm{~nm}$ and $1.6 \mu \mathrm{m}$ diameter lead to average $\mathrm{R}_{\mathrm{OFF}} / \mathrm{R}_{\mathrm{ON}}$ ratios of 50 and 2 , respectively. Moreover, no significant effect of layer thickness on the $\mathrm{R}_{\mathrm{OFF}} / \mathrm{R}_{\mathrm{ON}}$ ratio was noticed, as $\mathrm{R}_{\mathrm{OFF}}$ and $\mathrm{R}_{\mathrm{ON}}$ values increase in the same proportions with film thickness. This scaling of $\mathrm{R}_{\mathrm{OFF}} / \mathrm{R}_{\mathrm{ON}}$ ratio with $1 / \mathrm{S}$ is conform to previous observations on $\mathrm{GaV}_{4} \mathrm{~S}_{8}$ thin films [4] and is consistent with the quasi-dissolution of a granular metallic filament in a Mott insulating matrix. Higher $\mathrm{R}_{\mathrm{OFF}} / \mathrm{R}_{\mathrm{ON}}$ ratios are then expected for electrode sizes lower than $330 \mathrm{~nm}$. It must be noted that the resistive switching cycles obtained on a very thick layer (880nm, see Fig. 4(a)) without any initial forming definitely exclude any potential OxRAM mechanism, since the diffusion of oxygen vacancies on such distances is not realistic.

Temporal retention of OFF and ON resistance states presented in Fig. 4(b) shows a very good stability of both states at room temperature. The slight increase of $\mathrm{R}_{\mathrm{OFF}} / \mathrm{R}_{\mathrm{ON}}$ ratio with time 
(a)

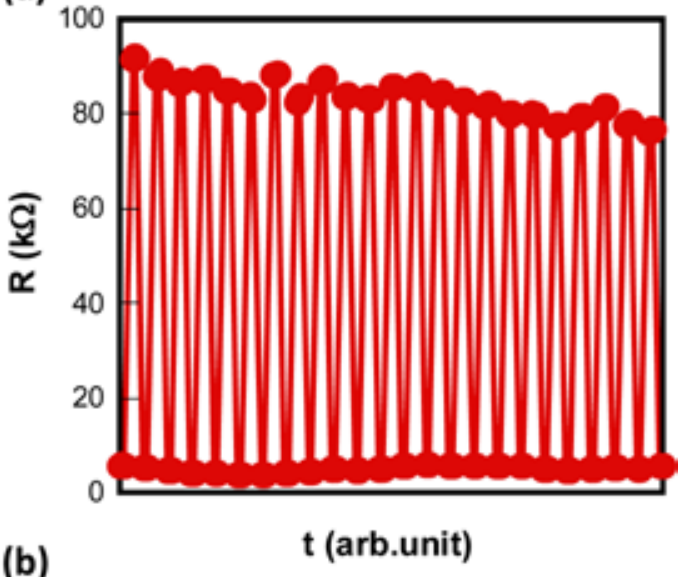

(b)

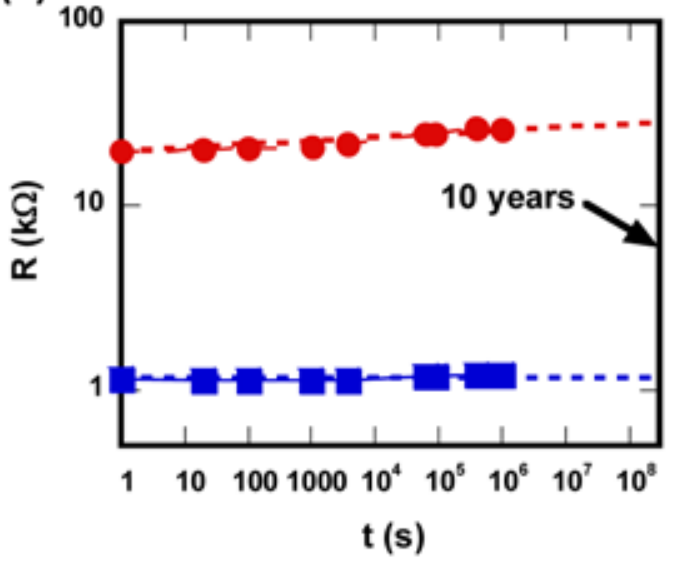

Fig.4: (a) Resistive switching cycles obtained at room temperature on devices with $880 \mathrm{~nm}$ thick films of $\left(\mathrm{V}_{0.95} \mathrm{Cr}_{0.05}\right)_{2} \mathrm{O}_{3}$ and $500 \mathrm{~nm}$ vias, by applying $3.5 \mathrm{~V} / 1.5 \mu$ s pulses for the SET and $4.5 \mathrm{~V} / 250 \mathrm{~ns}$ pulses for RESET. (b) Data retention at room temperature, extrapolated to 10 years, for OFF (red circles) and ON (blue squares) states on a $880 \mathrm{~nm}$ thick film of $\left(\mathrm{V}_{0.95} \mathrm{Cr}_{0.05}\right)_{2} \mathrm{O}_{3}$.

confirms previous measurements on $\mathrm{GaV}_{4} \mathrm{~S}_{8}$ thin films [2, 17]. It has to be noted that resistive switching cycles could be obtained on the device after 12 days of retention test. Lastly, preliminary endurance results show that these devices can be cycled more than 1000 times.

\section{Conclusions}

We have demonstrated non-volatile resistive switching in the canonical Mott insulator $\left(\mathrm{V}_{0.95} \mathrm{Cr}_{0.05}\right)_{2} \mathrm{O}_{3}$. Experiments conducted on crystals reveal that this resistive switching is consistent with the formation of granular conducting filaments under electric field. These filaments contain metallic domains closely related to the correlated metallic phase observed under high pressure in the P-T phase diagram of $\left(\mathrm{V}_{1-\mathrm{x}} \mathrm{Cr}_{\mathrm{x}}\right)_{2} \mathrm{O}_{3}$. It confirms therefore that the resistive switching in $\left(\mathrm{V}_{1-\mathrm{x}} \mathrm{Cr}_{\mathrm{x}}\right)_{2} \mathrm{O}_{3}$ is related to an electric field driven Mott transition at the local scale as observed previously in chalcogenide Mott insulators.

Memory cell devices based on the Mott insulator oxide $\left(\mathrm{V}_{0.95} \mathrm{Cr}_{0.05}\right)_{2} \mathrm{O}_{3}$ were fabricated with an excellent crystalline quality of the layers. The demonstrated performances are competitive in terms of programming voltage / time. Moreover the increase of $\mathrm{R}_{\mathrm{OFF}} / \mathrm{R}_{\mathrm{ON}}$ ratio with miniaturization, and the

preliminary retention / endurance tests are very promising. These results definitely set $\left(\mathrm{V}_{1-\mathrm{x}} \mathrm{Cr}_{\mathrm{x}}\right)_{2} \mathrm{O}_{3}$ based Mott memories as promising candidates among ReRAM solutions.

\section{References}

[1] F. Pan, S. Gao, C. Chen, C. Song, F. Zeng, Mater. Sci. Eng. R Rep. 83 (2014) 1-59.

[2] D.S. Jeong, R. Thomas, R.S. Katiyar, J.F. Scott, H. Kohlstedt, A. Petraru, C.S. Hwang, Rep. Prog. Phys. 75 (2012) 076502.

[3] R. Waser, M. Aono, Nat. Mater. 6 (2007) 833-840.

[4] E. Janod, J. Tranchant, B. Corraze, M. Querré, P. Stoliar, M. Rozenberg, T. Cren, D. Roditchev, V.T. Phuoc, M.-P. Besland, L. Cario, Adv. Funct. Mater. 25 (2015) 6287-6305.

[5] D.B. McWhan, J.P. Remeika, Phys. Rev. B 2 (1970) 3734-3750.

[6] L. Cario, C. Vaju, B. Corraze, V. Guiot, E. Janod, Adv. Mater. 22 (2010) 5193-5197.

[7] V. Guiot, L. Cario, E. Janod, B. Corraze, V. Ta Phuoc, M. Rozenberg, P. Stoliar, T. Cren, D. Roditchev, Nat. Commun. 4 (2013) 1722.

[8] P. Stoliar, P. Diener, J. Tranchant, B. Corraze, B. Brière, V. Ta-Phuoc, N. Bellec, M. Fourmigué, D. Lorcy, E. Janod, L. Cario, J. Phys. Chem. C 119 (2015) 2983-2988.

[9] P. Stoliar, L. Cario, E. Janod, B. Corraze, C. Guillot-Deudon, S. Salmon-Bourmand, V. Guiot, J. Tranchant, M. Rozenberg, Adv. Mater. 25 (2013) 3222-3226.

[10] V. Dubost, T. Cren, C. Vaju, L. Cario, B. Corraze, E. Janod, F. Debontridder, D. Roditchev, Adv. Funct. Mater. 19 (2009) 2800-2804.

[11] V. Dubost, T. Cren, C. Vaju, L. Cario, B. Corraze, E. Janod, F. Debontridder, D. Roditchev, Nano Lett. 13 (2013) 3648-3653.

[12] M. Querré, E. Janod, L. Cario, J. Tranchant, B. Corraze, V. Bouquet, S. Deputier, S. Cordier, M. Guilloux-Viry, M.-P. Besland, Thin Solid Films 617, Part B (2016) 56-62.

[13] Y. Ueda, K. Kosuge, S. Kachi, J. Solid State Chem. 31 (1980) 171-188.

[14] C. Vaju, L. Cario, B. Corraze, E. Janod, V. Dubost, T. Cren, D. Roditchev, D. Braithwaite, O. Chauvet, Adv. Mater. 20 (2008) 2760-2765.

[15] M.M. Abd-Elmeguid, B. Ni, D.I. Khomskii, R. Pocha, D. Johrendt, X. Wang, K. Syassen, Phys. Rev. Lett. 93 (2004).

[16] R. Pocha, D. Johrendt, B. Ni, M.M. Abd-Elmeguid, J. Am. Chem. Soc. 127 (2005) 8732-8740.

[17] D.B. McWhan, A. Menth, J.P. Remeika, W.F. Brinkman, T.M. Rice, Phys. Rev. B 7 (1973) 1920-1931.

[18] J. Tranchant, E. Janod, B. Corraze, P. Stoliar, M. Rozenberg, M.-P. Besland, L. Cario, Phys. Status Solidi A 212 (2015) 239-244.

[19] J. Tranchant, E. Janod, L. Cario, B. Corraze, E. Souchier, J.-L. Leclercq, P. Cremillieu, P. Moreau, M.-P. Besland, Thin Solid Films 533 (2013) 61-65.

Acknowledgements : The authors acknowledge SATT Ouest-Valorisation, CNRS and "Region Pays de la Loire" for funding. 